\title{
Blind Turbo Channel Estimation in OFDM Receivers
}

\author{
Thorsten Petermann, Sven Vogeler, \\ Karl-Dirk Kammeyer \\ Dept. of Communications Engineering \\ University of Bremen, P.O. Box 330440 \\ 28334 Bremen, Germany \\ \{petermann, vogeler, kammeyer\}@ant.uni-bremen.de
}

\author{
Dieter Boss
}

\author{
Research Dept. \\ Ericsson Eurolab \\ 90411 Nuremberg, Germany \\ Dieter.Boss@eed.ericsson.se
}

\begin{abstract}
Finite-Alphabet-based blind channel estimation in OFDM systems is known to be extremely complex due to an exhaustive search to be performed over a tremendous number of channel coefficient combinations. In this paper, we present a novel blind channel estimator which dramatically reduces this number of coefficient combinations to be checked without a significant deterioration in estimation quality. Hence, the new low complexity approach enables the application of blind channel estimators based on the finite alphabet set even if the transmitted data are high-rate modulated. Furthermore, we show that the performance of blind channel estimation can be improved by an iterative process based upon the capabilities of channel coding. Using bit error rates, the algorithm is tested with simulations and compared to other blind and nonblind channel estimators.
\end{abstract}

\section{Introduction}

In recent years, orthogonal frequency division multiplexing (OFDM) has become one of the most important techniques for high-rate wireless data transmission. Especially, it has been proposed for the European HIPERLAN/2 standard and the American equivalent IEEE802.11a; two similar concepts for broadband wireless local area networks (WLAN) in the $5 \mathrm{GHz}$ band. Since both standards include coherent data demodulation, the transmission channel has to be estimated. Generally, this is achieved by nonblind channel estimators exploiting additionally transmitted training data. Moreover, these training sequences have to be transmitted periodically, since the channel in wireless applications normally is time variant. In order to increase bandwidth efficiency, blind channel estimation, on the other hand, is well motivated since it avoids the need of any training data.

In recent publications, several blind channel estima- tion approaches based on second order cyclostationary statistics (SOCS) [3, 4] or subspace decompositions [5] have been tested for OFDM systems. Both classes of estimators are known to require long enough data records, in order to derive unbiased channel estimates. While subspace methods in general suffer from fading subcarriers (i.e., the channel has nulls on some subcarriers), the main drawback of algorithms based on SOCS is their sensibility towards "singular" channel classes with common subsystems (i.e. common zeros) in all polyphase subchannels. Furthermore, cyclostationary methods require some excess bandwidth.

In [1], the authors have demonstrated that it is possible with an eigenvector algorithm based on higher order statistics (HOS), referred to as EVI, to derive good channel estimates from fourth order cumulants estimated on the basis of only few samples of the received signal. In feasibility studies based on the global system for mobile communications (GSM), we have shown that the blind EVI algorithm can even compete with conventional second order methods based on training sequences [2]. It is well-known that cumulants of order three or higher vanish for Gaussian random processes. In other words, a Gaussian random process superimposed onto a non-Gaussian random process does not add anything to the higher order cumulants of said non-Gaussian process. This property is exploited by a multitude of signal processing algorithms based on HOS, where for instance a received signal is observed in additive Gaussian noise. However, due to the independence of subcarriers, in OFDM systems not only noise but also the transmitted signal is Gaussian distributed which means that HOS-based methods will fail under these circumstances.

The so-called Minimum Distance (MD) algorithm [8] is a blind estimator which is based on the knowledge that the modulated and transmitted data are 
confined to a finite alphabet (FA) set. However, it requires an enormous computational effort. In contrast, our novel Clustered SubCarriers (CSC) scheme dramatically reduces this effort without any significant deterioration in estimation quality. Furthermore, we can improve the performance of both blind and nonblind estimators with an iterative channel estimation scheme including the capabilities of channel coding.

The paper is organized as follows: Section 2 gives an overview of the OFDM system. In section 3, the principles of blind FA-based channel estimation are described. Exploiting the capabilities of channel coding, we present in section 4 the turbo channel estimation scheme. After showing some simulation results in section 5 , the paper is concluded in section 6 .

\section{Data transmission in OFDM systems}

Figure 1 shows the conventional OFDM system with Cyclic Prefix (CP). The CP of length $N_{c p}$ larger

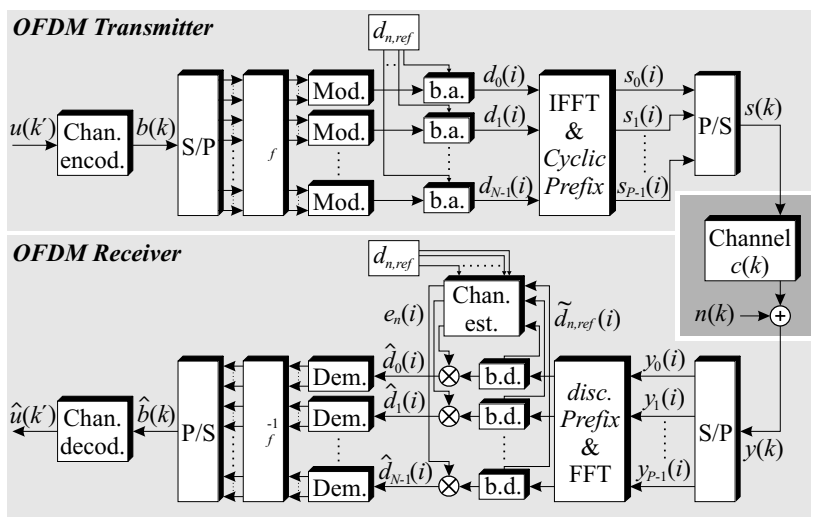

Figure 1: Conventional OFDM system with Cyclic Prefix

than the channel order $q$ avoids the received data from being disturbed by inter-symbol or inter-carrier interference (ISI/ICI). In the transmitter, the channel encoded information stream $b(k)$ is serial-to-parallel converted $(\mathrm{S} / \mathrm{P})$, interleaved $\left(\Pi_{f}\right)$, modulated, and assembled into the so-called OFDM symbols $\mathbf{d}(i):=$ $\left[d_{0}(i), d_{1}(i), \ldots, d_{N-1}(i)\right]^{T}$ of length $N$. After $\mathrm{P} / \mathrm{S}$ conversion, the OFDM sequence ${ }^{1} s(k)$ is transmitted over the time discrete channel $c(k)=\left(g_{c} * c_{c} *\right.$ $\left.h_{c}\right)\left.(t)\right|_{t=k T}$, where $*$ denotes convolution, $T$ is the chip period, and $g_{c}(t), h_{c}(t)$, and $c_{c}(t)$ are the time continuous transmit and receive filters and the physical channel, respectively. Under assumption that ICI and ISI have been prevented from occurring $\left(N_{c p}>q\right)$, the $i$ th received OFDM symbol $\tilde{\mathbf{d}}(i)$ after $\mathrm{S} / \mathrm{P}$ conversion

\footnotetext{
${ }^{1} k=i N+n, \quad n \in[0, N-1] \quad$ defines the chip index, where $n$ is the subcarrier index in frequency domain and $i$ characterizes the OFDM symbol index in time domain.
}

is calculated by

$$
\tilde{\mathbf{d}}(i)=\mathbf{D}_{C} \mathbf{d}(i)+\tilde{\mathbf{n}}(i),
$$

where $\mathbf{D}_{C}:=\operatorname{diag}\left[C\left(e^{j 0}\right), C\left(e^{j \frac{2 \pi}{N}}\right), \ldots, C\left(e^{j \frac{2 \pi}{N}(N-1)}\right)\right]$ is a diagonal matrix with diagonal elements $C\left(z_{n}\right):=$ $\sum_{l=0}^{q} c(l) z_{n}^{-l}$ evaluated at the subcarriers $z_{n}=e^{j \frac{2 \pi}{N} n}$ for each $n \in[0, N-1]$ and $\tilde{\mathbf{n}}(i)$ represents Additive Gaussian Noise (AGN) without $\mathrm{CP}$ and colored by the receive filter $h_{c}(t)$. From (1) it is obvious that the channel influence is reduced to one complex Rayleigh fading factor (channel coefficient) on each subcarrier.

Since each OFDM symbol has to be demodulated coherently, the channel coefficients $C(n):=C\left(e^{j \frac{2 \pi}{N} n}\right)$ have to be estimated. Therefore, let $\hat{C}(n)$ denote the estimate of each subcarrier $n$ which will be used to equalize $\tilde{d}_{n}(i)$

$$
\hat{\mathbf{d}}(i)=\mathbf{D}_{e} \tilde{\mathbf{d}}(i)
$$

where $\mathbf{D}_{e}:=\operatorname{diag}\left[e_{0}(i), e_{1}(i), \ldots, e_{N-1}(i)\right]$ with equalizer coefficients $e_{n}(i)=1 / \hat{C}(n)$. Finally, each OFDM symbol is de-interleaved $\left(\Pi_{f}^{-1}\right), \mathrm{P} / \mathrm{S}$ converted, and channel decoded into bits $\hat{u}\left(k^{\prime}\right)$. If channel decoding is based on soft values, it is important that the demodulated bits of each subcarrier $n$ are multiplied with the channel state information (CSI) $|\hat{C}(n)|^{2}$ before de-interleaving (not shown in Fig. 1, see Fig. 3).

\section{Blind FA-based channel estimation}

According to the PHY layer of HIPERLAN/2 and IEEE802.11a, several OFDM symbols are combined to bursts of different lengths $I$. In case of nonblind channel estimation, each burst is preceded by a preamble consisting of two identical training symbols $d_{n, \text { ref }}$. In general, the estimated channel transfer coefficient $\hat{C}(n)$ is disturbed by AGN. By exploiting the correlations between adjacent subcarrier coefficients and taking into account that the channel impulse response does not violate the $\mathrm{CP}$, a noise reduction can further improve estimation quality [6].

If the channel shall be estimated blindly, a burst only contains information-bearing symbols increasing the bandwidth efficiency. In [9], Zhou et al. have proven that for any $M$-ary modulation ${ }^{2}$ there can be found a variable $J \leq M$ (and for large signal constellations, $J \ll M)$ which is sufficient to eliminate the phase information of the received OFDM symbols: $\tilde{d}_{n}^{J}(i)=C^{J}(n) \cdot d_{n}^{J}(i)$. Concerning the complexity of FA-based blind channel estimators, this fact plays a very important role.

\footnotetext{
${ }^{2}$ For BPSK (Binary Phase Shift Keying), QPSK (Quadrature Phase Shift Keying), and 16-QAM (Quadrature Amplitude Modulation) $M=2,4$, and 16 , respectively.
} 
With $\mathrm{E}\left\{\tilde{d}_{n}^{J}(i)\right\}=C^{J}(n) \mathrm{E}\left\{d_{n}^{J}(i)\right\}$, each subcarrier coefficient is estimated by time averaging over $I$ OFDM symbols

$$
\hat{C}^{J}(n)=a \cdot \frac{1}{I} \sum_{i=0}^{I-1} \tilde{d}_{n}^{J}(i) \quad n \in[0, N-1],
$$

where $\mathrm{E}\{\cdot\}$ denotes expectation value and $a=$ $\mathrm{E}\left\{d_{n}^{J}(i)\right\} \neq 0$ is a real valued constant whose calculation is described in [9]. Since the colored noise $\tilde{n}_{n}(i)$ is zero-mean, eq. (3) also holds in the noisy case.

With respect to (3), MD [8] has to search over $J^{N}$ possible channel coefficient combinations $\hat{\mathbf{C}}_{1}:=\left[\lambda_{0}\left[\hat{C}^{J}(0)\right]^{1 / J}, \ldots, \lambda_{N-1}\left[\hat{C}^{J}(N-1)\right]^{1 / J}\right]^{T}$, where $\lambda_{n} \in\left\{e^{j \frac{2 \pi}{J} m}\right\}_{m=0}^{J-1}$ is a scalar ambiguity corresponding to the $J$ th root, $\forall n$. However, for QPSK modulated signals $(J=4)$ transmitted over a HIPERLAN/2 or IEEE802.11a channel with $N=52$ active subcarriers this means that $J^{N} \approx 2 \cdot 10^{31}$. Therefore, Zhou and Giannakis have presented a complexityreduced version of MD, the so-called Modified Minimum Distance algorithm (MMD) [8], which elleviates the effort to $J^{q+1} \ll J^{N}$, since, in general, the channel order $q \ll N$. Nevertheless, this can be still quite complex for typical HIPERLAN/2 channels. Our new CSC approach is mainly based on the idea of MD, but dramatically reduces the computational effort irrespective of the number of subcarriers or the channel order so that it will be possible to apply a FA-based blind channel estimator even to high-rate modulated OFDM systems.

\subsection{Clustered subcarriers algorithm}

Figure 2 shows the magnitude and phase of a typical HIPERLAN/2 transfer function, where $N=64$. The lower subplot depicts a steady
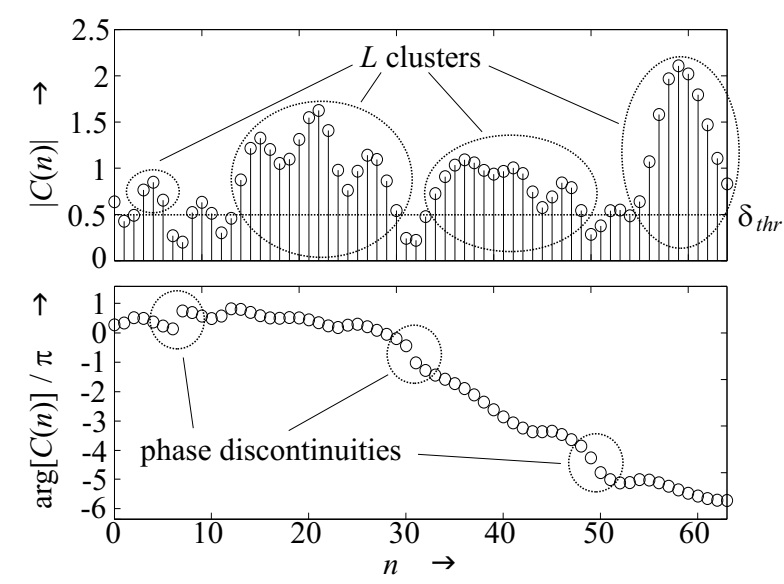

Figure 2: Magnitude and phase of a channel transfer function

phase course, except from the fading subcarriers $n \in\{6,7,30,31,49,50\}$, where phase discontinuities are obvious (dotted circles). Hence, it must be possible to track the scalar ambiguity $\lambda_{n}$ of adjacent channel coefficients by choosing their minimum phase distances. This assumption is true as long as no phase discontinuities appear. Therefore, we separated the transfer function into $L$ clusters consisting of $\ell_{\nu}, \nu \in[0, L-1]$, adjacent strong channel coefficients, whose magnitudes are above a certain threshold $\delta_{t h r}$. Within these clusters, phase discontinuities are very unlikely. By exploiting the correlation between the adjacent channel coefficients, their minimum phase distances within each cluster $\nu$ are searched and the scalar ambiguity factors

$$
\begin{gathered}
\lambda_{\nu, \mu}=\arg \min _{\lambda}\left|\hat{C}_{c l}(\nu, \mu-1)-\lambda\left[\hat{C}^{J}(\nu, \mu)\right]^{1 / J}\right|, \\
\mu \in\left[1, \ell_{\nu}-1\right], \quad \lambda_{\nu, 0}=1
\end{gathered}
$$

can be tracked from one coefficient to the other, where $\hat{C}_{c l}(\nu, \mu)=\lambda_{\nu, \mu}\left[\hat{C}^{J}(\nu, \mu)\right]^{1 / J}$. Finally, we collect $\hat{C}_{c l}(\nu, \mu)$ in $\ell_{\nu} \times 1$ cluster vectors $\hat{\mathbf{C}}_{c l, \nu}=$ $\left[\hat{C}_{c l}(\nu, 0), \ldots, \hat{C}_{c l}\left(\nu, \ell_{\nu}-1\right)\right]^{T}$ each containing only one scalar ambiguity. Thus, the remaining ambiguities can be resolved by searching over $J^{L} \ll J^{N}$ possible vectors $\hat{\mathbf{C}}_{2}=\left[\lambda_{0} \hat{\mathbf{C}}_{c l, 0}, \ldots, \lambda_{L-1} \hat{\mathbf{C}}_{c l, L-1}\right]^{T}$. This means that the computational effort of CSC does not depend anymore on the total number of subcarriers. On the contrary, it rather profits from as much as possible subcarriers, since each cluster may contain more correlated coefficients.

It must be mentioned that with a misadjusted threshold $\delta_{t h r}$, CSC might not correctly estimate $C(n)$ when phase discontinuities appear within clusters. Furthermore, all blind estimators come with an inherent remaining overall scalar ambiguity. This problem can only be solved by the aid of pilot carriers [8].

\section{Turbo channel estimation}

Figure 3 shows the concept of iterative channel estimation within an OFDM receiver. With respect to

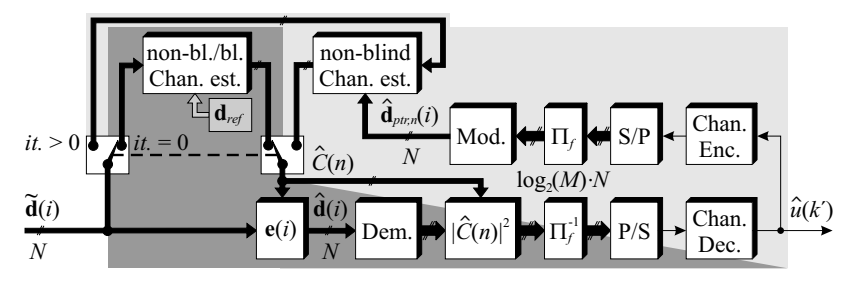

Figure 3: OFDM Turbo Channel Estimation

(2), the OFDM symbols $\tilde{\mathbf{d}}(i)$ after CP discarding and FFT transformation are equalized by means of $\mathbf{e}(i)$. 
a) Bit error rates after channel decod.

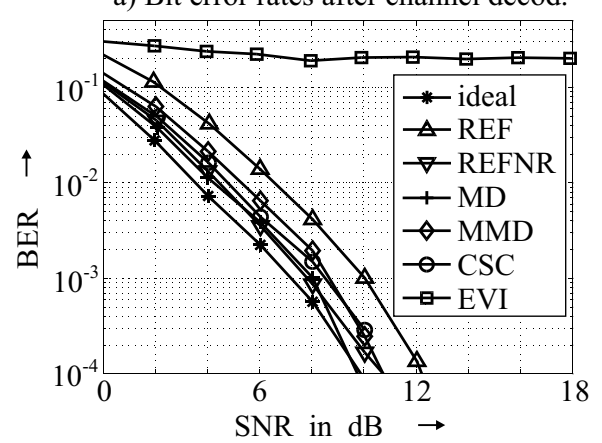

b) Computational effort (FA)

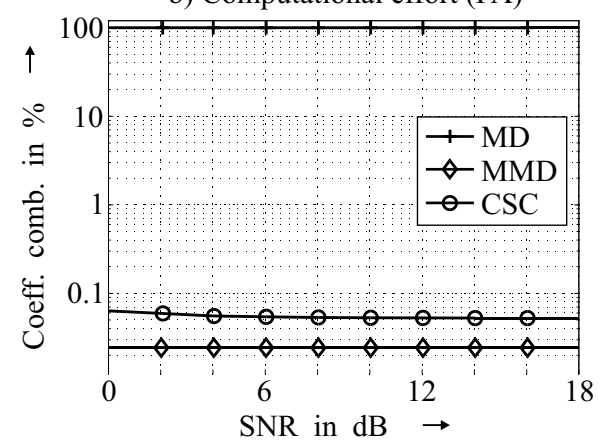

Figure 4: a) BERs of BPSK modulated symbols $(M=2, J=2)$ after "ideal", nonblind (REF, REFNR), and blind (MD, MMD, CSC, EVI) estimation of a 4-path Rayleigh fading channel, where $N=16, N_{c p}=6$, and $q=3$. b) Computational effort of the three blind FA-based estimators MD, MMD, and CSC.

Let us first consider the initial step of channel estimation $(i t .=0)$ characterized by the dark grey box and the two switches set to their inner position. According to section 3, e $(i)$ can be based on nonblind or blind channel estimates $\hat{C}(n)$, where in the nonblind case only the first two OFDM symbols of each burst are utilized for estimation, while blind estimates are based on the complete burst consisting of $I$ OFDM symbols. After demodulation, each $\log _{2}(M)$ bits have to be multiplied with the CSI $|\hat{C}(n)|^{2}$ before de-interleaving, if the following channel decoder is based on soft values. Now, the process of iteration (characterized by the light grey box and both switches set to their outer position, it. $>0)$ starts with re-encoding of the channel decoded bits $\hat{u}\left(k^{\prime}\right)$. Upon $\mathrm{S} / \mathrm{P}$ conversion, interleaving, and modulation, the new pseudo training OFDM symbol $\hat{\mathbf{d}}_{p t r}(i)$ can be utilized by a nonblind channel estimator

$$
\hat{C}(n)=\frac{1}{I} \sum_{i=0}^{I-1} \frac{\tilde{d}_{n}(i)}{\hat{d}_{p t r, n}(i)} .
$$

On the one hand, time averaging over the burst length $I$ might impair the estimation of time variant channels, but, on the other hand, the influence of noise can be reduced significantly. Finally, the estimation performance can be improved by an additional noise reduction.

\section{Simulation results}

In this section, we compare the influence of blind and nonblind channel estimators on the equalization of the received data through MONTE-CARLO simulations. With regard to section 2, bursts of $M$-ary modulated OFDM symbols of length $N$ were transmitted over different time invariant ${ }^{3}$ channels of order

\footnotetext{
${ }^{3}$ The channel coefficients were changed from burst to burst so that the channel is assumed to be time invariant only over one burst period.
}

$q$ for signal-to-noise ratios (SNRs) ranging from 0 to $18 \mathrm{~dB}$. In the nonblind case, each burst is preceded by two identical BPSK modulated training symbols, while blind estimators exploit the complete burst consisting of $I$ information bearing symbols. By comparing the sequences $\hat{u}\left(k^{\prime}\right)$ and $u\left(k^{\prime}\right)$, bit error rates (BERs) were calculated after channel decoding (see Figs. 1 and 3), where a half-rate convolutional code with constraint length $L_{c}=5$ was applied.

Figure 4-(a) shows the BERs of BPSK modulated OFDM symbols $(M=2, J=2)$ transmitted over a Rayleigh fading channel of order $q=3$ with $N=16$ subcarriers and CP length $N_{c p}=6$ for an "ideal", nonblind (REF, REFNR), blind FAbased (MD, MMD, CSC), and HOS-based (EVI) channel estimation. Subplot (b) illustrates the computational effort of the three blind FA-based algorithms, where the estimator with highest complexity was set to $100 \%$. Let us first compare the two nonblind estimators. From subplot (a) it is obvious that with an additional noise reduction REFNR gains app. $2 \mathrm{~dB}$ in SNR over REF and nearly reaches the estimator with "ideal" channel knowledge. On the other hand, we can see that the blind MD approach shows the same excellent estimation performance as REFNR. Even the estimation quality of the two blind estimators with reduced complexity, MMD and our novel CSC, is almost comparable to that of MD. This is very remarkable, since according to subplot (b) both CSC and MMD have to check about more than 1000 times less channel coefficient combinations than MD. Finally, we can see that EVI fails in OFDM systems due to vanishing cumulants caused by the Gaussianity of the transmitted signals.

Figure 5 depicts the BERs of QPSK modulated OFDM symbols $(M=4, J=4)$, where, according to the ETSI-BRAN project [7], a time invariant HIPER- 
a) Burst length $I=20$

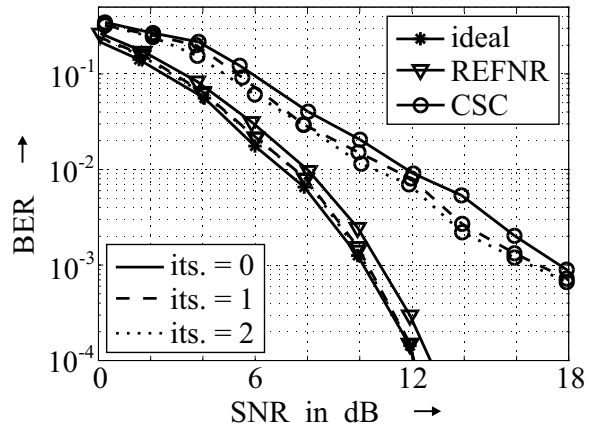

b) Burst length $I=200$

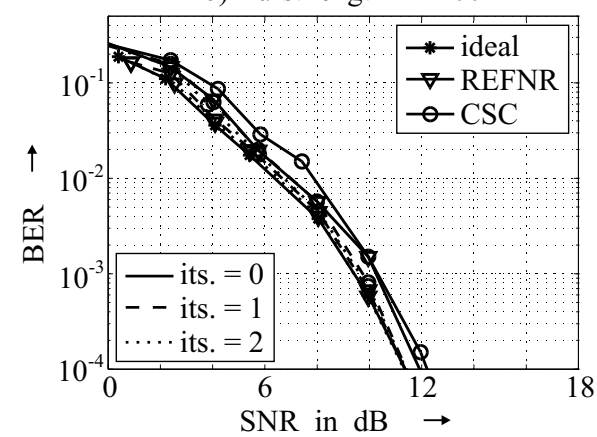

Figure 5: BERs of QPSK modulated symbols $(M=4, J=4)$ after turbo estimation of a HIPERLAN $/ 2$ type A channel $(q=8)$ with 0,1 , and 2 iterations for an initial "ideal", nonblind (REFNR), and blind (CSC) estimator, where $N=52, N_{c p}=16$, and the burst lengths are set to $I=20$ (a) and $I=200$ OFDM symbols (b).

LAN/2 type A channel of order $q=8$ with $N=52$ subcarriers and a CP length of $N_{c p}=16$ was used. While for subplot (a) each burst consisted of $I=20$ OFDM symbols, in subplot (b) the burst length was set to $I=200$. Furthermore, a turbo channel estimation scheme with two iterations was applied after an initial "ideal", nonblind (REFNR), and blind (CSC) estimation. Due to the enormous number of channel coefficient combinations to be checked in this scenario $\left(J^{N}=4^{52}\right)$, the investigation of MD was impossible. With $J^{q+1}=4^{9}$, even the computational effort of MMD was too high so that it could not be tested here. If we compare the BERs after initial channel estimation (its. $=0$, solid lines), it can be noticed from subplot (a) that with a burst length of $I=20$ OFDM symbols, at $\mathrm{SNR}=10^{-3}$, CSC shows a SNR loss of app. $7 \mathrm{~dB}$ compared to REFNR while, with regard to subplot (b), CSC almost reaches REFNR when $I=200$. This can be explained by a more accurate estimation of the pseudo reference (3) due to a larger amount of utilizable OFDM symbols. Finally, subplot (b) shows that with a burst length of $I=200$ and two iterations, the application of the turbo scheme to both an initial CSC and REFNR leads to the performance of the estimator with ideal channel knowledge.

\section{Conclusions}

In this paper, we have presented a novel low complexity blind channel estimation approach for OFDM related systems which distinguishes through the fact that it enables the application of Finite-Alphabetbased blind channel estimators even to high-rate modulated OFDM signals. Furthermore, a turbo channel estimation scheme was introduced which improves the quality of both blind and nonblind estimators by exploiting the capabilities of channel coding.

\section{References}

[1] D. Boss, B. Jelonnek, and K. Kammeyer. Eigenvector Algorithm for Blind MA System Identification. Elsevier Signal Processing, 66(1):1-26, April 1998.

[2] D. Boss, K. Kammeyer, and T. Petermann. Is Blind Channel Estimation feasible in Mobile Communication Systems? A Study based on GSM. IEEE Journal on Selected Areas in Communications, 16(8):1479-1492, October 1998. Special issue on Signal Processing for Wireless Communications.

[3] J. Heath and G. Giannakis. Exploiting Input Cyclostationarity for Blind Channel Identification in OFDM Systems. IEEE Trans. on Signal Processing, 47(3):848-856, March 1999.

[4] R. Klinski, H. Hutzelmann, and R. Knorr. Low Complexity Blind Channel Estimation for OFDM Systems. In Proc. WSES Multiconference on Circuits, Systems, Communications \& Computers (CSCC), Crete, Greece, July 2001.

[5] B. Muquet, M. de Courville, P. Duhamel, and V. Buenac. A Subspace Based Blind and Semi-Blind Channel Identification Method for OFDM Systems. In Proc. IEEE-SP Workshop on Signal Proc. Advances in Wireless Comm., pages 170-173, Annapolis, MD, USA, May 1999.

[6] H. Schmidt, V. Kühn, K. Kammeyer, R. Rückriem, and S. Fechtel. Channel Tracking in Wireless OFDM Systems. In Proc. Multi-Conference on Systemics, Cybernetics and Informatics, Orlando, Florida, USA, July 2001.

[7] P. Wijk. ETSI. Criteria of Comparison. Technical Report 30701F, ETSI EP BRAN WG3 meeting \#9, 1998.

[8] S. Zhou and G. Giannakis. Finite-Alphabet based Channel Estimation for OFDM and related MultiCarrier Systems. IEEE Trans. on Communications, 49, 2001.

[9] S. Zhou, G. Giannakis, and A. Scaglione. Long Codes for Generalized FH-OFDMA through Unknown Multipath Channels. IEEE Trans. on Communications, 2001. 